\title{
Semiexplicit $A$-Stable Runge-Kutta Methods
}

\author{
By G. J. Cooper and A. Sayfy
}

\begin{abstract}
An $s-1$ stage semiexplicit Runge-Kutta method is represented by an $s \times s$ real lower triangular matrix where the number of implicit stages is given by the number of nonzero diagonal elements. It is shown that the maximum order attainable is $s$ when $s \leqslant 5$. Necessary and sufficient conditions for $A$-stability are derived and it is shown that there must be $s-1$ implicit stages if the order is $s$ and $s \leqslant 5$. Examples are given for $s \leqslant 4$ where all the nonzero diagonal elements are equal. Additional problems arise when $s>4$; but when $s=6$, an $A$-stable method of order 5 is obtained. This method has five nonzero diagonal elements, and these elements are equal. Finally, a six stage $A$ stable method of order six is given. Again, this method has five nonzero (and equal) diagonal elements.
\end{abstract}

1. Introduction. Consider an initial value problem, for a system of ordinary differential equations, of the form $x^{\prime}=f(x), x\left(t_{0}\right)=x_{0}$. An $s-1$ stage, semiexplicit, Runge-Kutta method computes a sequence of approximations

$$
y_{r}^{(m)}=y_{s}^{(m-1)}+h \sum_{j=1}^{r} b_{r j} f\left(y_{j}^{(m)}\right), \quad r=1,2, \ldots, s, m=1,2,3, \ldots,
$$

where $y_{s}^{(m)}$ approximates $x\left(t_{0}+m h\right), m=0,1,2, \ldots$. Such a method may be represented by a real triangular matrix $B$, and described by an array

$$
\begin{array}{c|ccc}
c_{1} & b_{11} & & \\
c_{2} & b_{21} & b_{22} & \\
\cdot & \cdot & & \\
\cdot & \cdot & & \\
\cdot & \cdot & & \\
c_{s} & b_{s 1} & b_{s 2} & \cdot \cdot b_{s s .}
\end{array}
$$

where $c_{r}=b_{r 1}+b_{r 2}+\cdots+b_{r r}, r=1,2, \ldots, s$. It is assumed that $c_{s}=1$ so that all methods are of order $p \geqslant 1$, and it is also assumed that $s \geqslant 2$. The convention $b_{s s}$ $=0$ is not adopted, even though little is gained thereby. Indeed, in this article it is proved that, for $s \leqslant 5$, the maximum attainable order is $p=s$ and that there are such methods with $b_{s s}=0$.

Suppose that an $s-1$ stage, semiexplicit, Runge-Kutta method is applied to the scalar initial value problem $x^{\prime}=\lambda x$ with $x(0)=1$. Then

* Received January 31, 1978.

AMS (MOS) subject classifications (1970). Primary 65L05.

(c) 1979 American Mathematical Society 0025-571 8/79/0000-0055/\$05.00 


$$
y_{s}^{(m)}=\frac{p(\tau)}{q(\tau)} y_{s}^{(m-1)}, \quad \tau=h \lambda, m=1,2,3, \ldots,
$$

where $p(\tau)$ is a polynomial of degree $s-1$ and

$$
q(\tau)=\prod_{r=1}^{s}\left(1-\tau b_{r r}\right)=1-\beta_{1} \tau+\beta_{2} \tau^{2}-\cdots+(-1)^{s} \beta_{s} \tau^{s}
$$

The method is defined to be $A$-stable if $|q(\tau)|>|p(\tau)|$ for all $\tau$ with $\operatorname{Re} \tau<0$. Thus, for any $A$-stable method, all diagonal elements of $B$ must be nonnegative; and at least one diagonal element must be positive. The trapezoidal rule [4] is an $A$-stable, semiexplicit, Runge-Kutta method which may be represented by the array

$$
\begin{array}{l|ll}
0 & 0 & \\
1 & 1 / 2 & 1 / 2
\end{array}
$$

where $b_{s s} \neq 0$. This method is of order $p=s=2$.

In each step of a method, a set of $n$ nonlinear equations has to be solved for each nonzero diagonal element, so that $\boldsymbol{A}$-stable, semiexplicit, Runge-Kutta methods of order $p=s$, with a maximum number of zero diagonal elements, are required. When the nonlinear equations are solved by certain variants of the Newton-Raphson iteration, there is a significant computational advantage in using a method where all the nonzero diagonal elements are equal [2]. Methods of this type with $p=s \leqslant 4$ have been obtained by Norsett [5].

In this article, necessary and sufficient conditions for $\boldsymbol{A}$-stability are obtained. These conditions, which are similar to conditions obtained by Norsett [6], are used to show that when $p=s \leqslant 5$ an $A$-stable, semiexplicit Runge-Kutta method can have at most one zero diagonal element. Methods are obtained with $p=s=4$ with one zero diagonal element and all other diagonal elements equal. One of these methods is due to Norsett [5], and the other method is obtained by choosing $b_{s s} \neq 0$. When $p=s$ $=5$, it is not possible to obtain an $A$-stable method with one zero diagonal element and all other diagonal elements equal. However, it is possible to obtain such methods when $p=5$ and $s=6$. This is of little interest because an $A$-stable method with $p=6$ and $s=7$ is obtained with two diagonal elements zero and all other diagonal elements equal.

2. Attainable Order of Semiexplicit Methods. An $s-1$ semiexplicit Runge-Kutta method with $b_{s s} \neq 0$ is equivalent to an $s$ stage method of the form

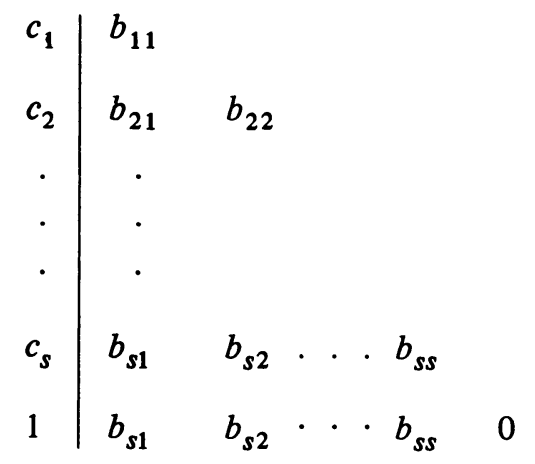


so that the order conditions may be obtained from those for conventional Runge-Kutta methods [1]. The conditions may also be obtained, directly, by adapting the treatment of Cooper and Verner [3]. It is convenient to state the conditions in terms of the expressions

$$
b_{r}(\sigma)=c_{r}^{\sigma}-\sigma \sum_{j=1}^{s} b_{r j} c_{j}^{\sigma-1}, \quad r=1,2, \ldots, s, \sigma=1,2,3, \ldots,
$$

where, as already defined, $b_{r}(1)=0$ for $r=1,2, \ldots, s$. Then the conditions

$$
\begin{gathered}
b_{s}(\sigma)=0, \quad \sigma \leqslant p, \\
\sum_{r=1}^{s} b_{s r} c_{r}^{\tau-1} b_{r}(\sigma)=0, \quad \sigma+\tau \leqslant p, \\
\sum_{r=1}^{s} b_{s r} c_{r}^{\tau-1} \sum_{j=1}^{s} b_{r j} c_{j}^{\nu-1} b_{j}(\sigma)=0, \quad \sigma+\tau+\nu \leqslant p, \\
\sum_{r=1}^{s} b_{s r} \sum_{j=1}^{s} b_{r j} \sum_{k=1}^{s} b_{j k} b_{k}(2)=0, \quad p \geqslant 5, \\
\sum_{r=1}^{s} b_{s r} b_{r}(2) b_{r}(2)=0, \quad p \geqslant 5,
\end{gathered}
$$

where $\sigma, \tau$ and $\nu$ take all possible positive integer values, are necessary for a method to be of order $p$, and are necessary and sufficient for a method to be of order $p \leqslant 5$.

It has been remarked that methods may be obtained with $b_{s s}=0$ and $p=s$ for $s \leqslant 5$. Consider the case $p=5$ and assume that $b_{s s}=0$. Then the order conditions may be replaced by the more restrictive conditions

$$
\begin{gathered}
b_{s}(\sigma)=0, \quad \sigma \leqslant 5, \\
b_{r}(2)=0, \quad r=1,2, \ldots, s-1, \\
\sum_{r=1}^{s} b_{s r} b_{r j}=b_{s j}\left(1-c_{j}\right), \quad j=3,4, \ldots, s-1, \\
\sum_{r=1}^{s} b_{s r} c_{r} b_{r j}=\frac{b_{s j}}{2}\left(1-c_{j}^{2}\right), \quad j=3,4, \ldots, s-1,
\end{gathered}
$$

which give a one parameter family of methods when $s=5$. In particular, the method 


\begin{tabular}{|c|c|c|c|c|}
\hline 0 & 0 & & & \\
\hline $5-\sqrt{ } 5$ & $5-\sqrt{ } 5$ & $5-\sqrt{ } 5$ & & \\
\hline 10 & 20 & 20 & & \\
\hline $5+\sqrt{ } 5$ & & $5+3 \sqrt{ } 5$ & $5-\sqrt{ } 5$ & \\
\hline 10 & 0 & 20 & 20 & \\
\hline 1 & $\frac{-1+\sqrt{ } 5}{4}$ & 0 & $\frac{5-\sqrt{ } 5}{4}$ & 0 \\
\hline 1 & $\frac{1}{12}$ & $\frac{5}{12}$ & $\frac{5}{12}$ & $\frac{1}{12}$ \\
\hline
\end{tabular}

has equal nonzero diagonal elements (though it is not $A$-stable).

THEOREM 1. When $s \leqslant 5$ the order of an $s-1$ stage semiexplicit Runge-Kutta method cannot exceed s.

Proof. Only the case $s=5$ is considered since the case $s=4$ may be proved by a similar argument and the other cases are comparatively trivial. It is shown that, when $s=5$ and $p=6$, the order conditions must lead to a contradiction.

Condition (1.1) gives, for any polynomial $\Pi$ of degree at most 5 ,

$$
\int_{0}^{1} \Pi(t) d t=\sum_{r=1}^{5} b_{5 r} \Pi\left(c_{r}\right) .
$$

Suppose there are at most two distinct values $c_{j}$ and $c_{k}$ in $\left\{c_{1}, c_{2}, c_{3}, c_{4}\right\}$. Then

$$
\begin{aligned}
& -\int_{0}^{1}\left(t-c_{j}\right)\left(t-c_{k}\right)(t-1) t^{\nu} d t=0 \\
& \quad=\frac{1}{(4+\nu)(3+\nu)}-\frac{c_{j}+c_{k}}{(3+\nu)(2+\nu)}+\frac{c_{j} c_{k}}{(2+\nu)(1+\nu)}, \quad v=0,1,2,
\end{aligned}
$$

gives a contradiction. Hence, at most, two values in $\left\{c_{1}, c_{2}, c_{3}, c_{4}\right\}$ are equal. Suppose $c_{j}=c_{k}$ with $j \neq k$. Then, $b_{5 j}+b_{5 k} \neq 0$; and $b_{5 r} \neq 0$ for $r \neq j, k$ and $r<5$.

If $c_{1}, c_{2}, c_{3}, c_{4}$ are distinct, condition (1.2) gives $b_{5 r} b_{r}(2)=0$ for all $r \leqslant 5$. If not, then $b_{5 r} b_{r}(2)=0$ for all $r \neq j, k$, where $c_{j}=c_{k}$ and (1.2) and (1.5) give

$$
b_{5 j} b_{j}(2)+b_{5 k} b_{k}(2)=0=b_{5 j} b_{j}(2)^{2}+b_{5 k} b_{k}(2)^{2}, \quad b_{5 j}+b_{5 k} \neq 0 .
$$

Hence, $b_{5 r} b_{r}(2)=0$ for all $r \leqslant 5$ and, in particular, $b_{51} c_{1}=0$.

Suppose $c_{2}, c_{3}, c_{4}$ are not distinct with $c_{j}=c_{k}, j \neq k$. Let $c_{r}$ be the other value. Then, since $b_{51} c_{1}=0$, condition (1.1) gives

$$
\begin{aligned}
& -\int_{0}^{1}\left(t-c_{j}\right)\left(t-c_{r}\right)(t-1) t^{\nu} d t=0 \\
& \quad=\frac{1}{(4+\nu)(3+\nu)}-\frac{c_{j}+c_{r}}{(3+\nu)(2+\nu)}+\frac{c_{j} c_{r}}{(2+\nu)(1+\nu)}, \quad v=1,2,
\end{aligned}
$$

whence $c_{r}=(4 \pm \sqrt{ } 6) / 10$ and $c_{j}=(4 \mp \sqrt{ } 6) / 10$. This gives $b_{5}(6) \neq 0$ so that $c_{2}, c_{3}$, $c_{4}$ must be distinct and $b_{5 r} c_{r} \neq 0$ for $r=2,3,4$. 
Now $b_{5 r} b_{r}(2)=0$ for all $r$ and $b_{51} c_{1}=0$ so that (1.3) gives

$$
b_{54} c_{4}^{\nu} \sum_{r=1}^{5} b_{4 r} b_{r}(2)+b_{53} c_{3}^{\nu} \sum_{r=1}^{5} b_{3 r} b_{r}(2)+b_{52} c_{2}^{\nu} b_{2 r} b_{1}(2)=0, \quad \nu=0,1,2 .
$$

Since $c_{2}, c_{3}, c_{4}$ are distinct $b_{52} b_{21} b_{1}(2)=0$. But $b_{52} \neq 0$ so that $b_{21} c_{1}=0$ and, also, $b_{2}(2)=0$. Thus, $b_{2}(3) \neq 0$. However, $(1.2)$ gives $b_{2}(3)=0$, a contradiction.

It has been mentioned that it is possible to obtain $A$-stable, semiexplicit, methods with $p=s=4$ which have equal nonzero diagonal elements. It is now shown that it is only possible to obtain a semiexplicit method of order $p=4$ of the form

$$
\begin{array}{l|llll}
c & c & & & \\
c_{2} & b_{21} & c & & \\
c_{3} & b_{31} & b_{32} & c & \\
1 & b_{41} & b_{42} & b_{43} & 0
\end{array}
$$

if $\left(24 c^{3}-36 c^{2}+12 c-1\right)\left(6 c^{2}-8 c+1\right)=0$. Since (1.1) gives a quadrature rule which is exact for any polynomial of degree three,

$$
\begin{aligned}
& -\int_{0}^{1}(t-c)\left(t-c_{2}\right)\left(t-c_{3}\right) d t=0 \\
& \quad=c c_{2} c_{3}-\frac{1}{2}\left(c c_{2}+c c_{3}+c_{2} c_{3}\right)+\frac{1}{3}\left(c+c_{2}+c_{3}\right)-\frac{1}{4} .
\end{aligned}
$$

Suppose that (1.1) holds. Then conditions (1.2) reduce to

$$
\begin{gathered}
b_{43} b_{32} c_{2}+\left(b_{43} b_{31}+b_{42} b_{21}\right) c=\frac{1-3 c}{6}, \\
b_{43} b_{32} c_{2}^{2}+\left(b_{43} b_{31}+b_{42} b_{21}\right) c^{2}=\frac{1-4 c}{12}, \\
b_{43} b_{32} c_{2} c_{3}+\left(b_{43} b_{31} c_{3}+b_{42} b_{41} c_{2}\right) c=\frac{3-8 c}{24},
\end{gathered}
$$

while condition (1.3) may be expressed as

$$
b_{43} b_{32}\left(c_{2}^{2}-4 c c_{2}+2 c^{2}\right)-\left(b_{43} b_{31}+b_{42} b_{21}\right) c^{2}=0 .
$$

It follows that $c \neq 0$ and that Eqs. (3.2), (3.3) and (3.5) can be solved only if $c_{2}(2 c-1)(6 c-1)=2 c\left(6 c^{2}-6 c+1\right)$ whence $2 c-1 \neq 0$. The equations give

$$
b_{43} b_{32}\left(c_{2}-c\right)^{2}=\frac{1-4 c}{24}, \quad b_{43} b_{32} c_{2}\left(c_{2}-c\right)=\frac{6 c^{2}-6 c+1}{12},
$$

so that $\left(c_{2}-c\right)(1-4 c) \neq 0$. Since $b_{21}=c_{2}-c$ and $b_{31}+b_{32}=c_{3}-c,(3.4)$ gives

$$
\frac{3-8 c}{24}=b_{43} b_{32} c_{3}\left(c_{2}-c\right)+b_{43} c c_{3}\left(c_{3}-c\right)+b_{42} c c_{2}\left(c_{2}-c\right) \text {. }
$$

Conditions (1.1) imply that

$$
b_{43} c c_{3}\left(c_{3}-c\right)+b_{42} c c_{2}\left(c_{2}-c\right)=\frac{c(2-3 c)}{6}
$$

and substitution gives $c_{3}(1-4 c)=\left(c_{2}-c\right)\left(12 c^{2}-16 c+3\right)$, whence 


$$
c_{3}=\frac{c\left(12 c^{2}-16 c+3\right)}{(2 c-1)(6 c-1)} \text {. }
$$

From (3.1), if $6 c c_{2}-3\left(c+c_{2}\right)+2=0$, then $6 c^{2}-6 c+1=0$ so that $c_{2}=0$, which gives a contradiction. Thus,

$$
c_{3}=\frac{6 c c_{2}-4\left(c+c_{2}\right)+3}{2\left\{6 c c_{2}-3\left(c+c_{2}\right)+2\right\}}=\frac{72 c^{4}-168 c^{3}+128 c^{2}-36 c+3}{2\left\{72 c^{4}-144 c^{3}+96 c^{2}-25 c+2\right\}}
$$

Hence a polynomial equation is obtained for $c$ and this factors as

$$
(2 c-1)^{2}\left(24 c^{3}-36 c^{2}+12 c-1\right)\left(6 c^{2}-8 c+1\right)=0 .
$$

The roots of $24 c^{3}-36 c^{2}+12 c-1$ are real and positive and give the methods

$$
\begin{array}{c|cccc}
c & c & & & \\
1 / 2 & \frac{1-2 c}{2} & c & & \\
1-c & 2 c & 1-4 c & c & \\
1 & \frac{2 c(c-1)}{2 c-1} & \frac{6 c-1}{6 c(2 c-1)} & \frac{2 c(c-1)}{2 c-1} & 0
\end{array}
$$

which were obtained by Norsett [4] who showed that one of these methods is $A$-stable. The roots of $6 c^{2}-8 c+1$ give the methods

$$
\begin{array}{c|cccc}
c & c & & \\
\frac{2}{3} & \frac{2-3 c}{3} & c & \\
\frac{1}{6 c} & \frac{4 c-1}{6 c} & \frac{1-4 c}{2 c} & c & \\
1 & \frac{1}{20 c} & \frac{3}{5} & \frac{3 c}{10} & 0
\end{array}
$$

though neither of these methods is $A$-stable.

3. Conditions for $A$-Stability. Suppose that an $s-1$ stage, semiexplicit, RungeKutta method

$$
\begin{array}{c|cc}
c_{1} & b_{11} & \\
c_{2} & b_{21} & b_{22} \\
\cdot & \cdot & \\
\cdot & \cdot & \\
\cdot & \cdot & \\
c_{s} & b_{s 1} & b_{s 2} \cdots \cdot b_{s s}
\end{array}
$$

is applied to the scalar initial value problem $x^{\prime}=\lambda x$ with $x(0)=1$. Then 


$$
y_{s}^{(m)}=\frac{p(\tau)}{q(\tau)} y_{s}^{(m-1)}, \quad \tau=h \lambda, m=1,2,3, \ldots,
$$

where $p$ is a polynomial of degree $s-1$ and $q$ a polynomial of degree $s$,

$$
\begin{aligned}
& p(\tau)=1-\alpha_{1} \tau+\alpha_{2} \tau^{2}-\cdots+(-1)^{s-1} \alpha_{s-1} \tau^{s-1} \\
& q(\tau)=\prod_{r=1}^{s}\left(1-\tau b_{r r}\right)=1-\beta_{1} \tau+\beta_{2} \tau^{2}-\cdots+(-1)^{s} \beta_{s} \tau^{s}
\end{aligned}
$$

The method is $A$-stable if $|q(\tau)|>|p(\tau)|$ for all $\tau$ with $\operatorname{Re} \tau<0$ so that it is necessary and sufficient that $b_{r r} \geqslant 0$ for $r=1,2, \ldots, s$ and $p(\tau) / q(\tau)<1, \operatorname{Re} \tau<0$. Since all methods are assumed to be of order $p \geqslant 1$ the rational function is not constant. As $q(\tau)$ is analytic for $\operatorname{Re} \tau \leqslant 0$, it follows from the maximum modulus principle that necessary and sufficient conditions for $A$-stability are that $b_{r r} \geqslant 0$ for $r=1,2, \ldots, s$ and $|p(i y)|^{2} \leqslant|q(i y)|^{2} \forall y \in R$. Now define $\beta_{0}=1$ and $\beta_{s+1}=\beta_{s+2}=\cdots=0$. Then

$$
\begin{aligned}
|q(\tau)|^{2} & =q(\tau) q(\bar{\tau})=\sum_{r=0}^{2 s}(-1)^{r} \sum_{j=0}^{r} \beta_{r-j} \beta_{j} \tau^{r-j \bar{\tau}^{j}} \\
& =\sum_{r=0}^{2 s}(-1)^{r} \sum_{j=0}^{[r / 2]} * \beta_{r-j} \beta_{j}\left(\tau^{r-j} \bar{\tau}^{j}+\tau^{j} \bar{\tau}^{r-j}\right)
\end{aligned}
$$

where $[\nu]$ is the integral part of $\nu$ and where the asterisk denotes that any term with $2 j=r$ is halved. It follows that

$$
|q(i y)|^{2}=2 \sum_{r=0}^{s} y^{2 r} \sum_{j=0}^{r} \beta_{2 r-j} \beta_{j}(-1)^{r+j}
$$

and there is a similar expressions for $|p(i y)|^{2}$ with $\alpha_{0}=1$ and $\alpha_{s}=\alpha_{s+1}=\cdots=0$. Hence an $s-1$ stage semiexplicit Runge-Kutta method is $A$-stable if and only if $b_{r r}=$ $0, r=1,2, \ldots, s$, and

$$
\sum_{r=1}^{s} y^{2 r} \sum_{j=0}^{r}\left(\beta_{2 r-j} \beta_{j}-\alpha_{2 r-j} \alpha_{j}\right)(-1)^{r+j} \geqslant 0 \quad \forall y \geqslant 0 .
$$

This result may be further simplified if it is assumed that the method is of order $p$ for then

$$
\left|\frac{p(\tau)}{q(\tau)}\right|^{2}=e^{\tau+\bar{\tau}}+O\left(|\tau|^{p+1}\right)
$$

whence $|q(i y)|^{2}-|p(i y)|^{2}=O\left(y^{p+1}\right)$. This gives the following result.

TheOREM 2. An $s-1$ stage semiexplicit Runge-Kutta method of order $p$ is $A$ stable if and only if $b_{r r} \geqslant 0, r=1,2, \ldots, s$, and

$$
\sum_{r=[(p+2) / 2]}^{s} y^{r} \sum_{j=0}^{r} *\left(\beta_{2 r-j} \beta_{j}-\alpha_{2 r-j} \alpha_{j}\right)(-1)^{r+j} \geqslant 0 \quad \forall y \geqslant 0 .
$$

This is similar to a result obtained by Norsett [6]. Other stability regions may also be examined. 
Suppose that an $s-1$ stage semiexplicit Runge-Kutta method is of order $p$. Then, since $p(\tau) / q(\tau)=c^{\tau}+O\left(|\tau|^{p+1}\right)$, it follows that

$$
\alpha_{r}=\beta_{r}-\beta_{r-1}+1 / 2 \beta_{r-2}-\cdots+(-1)^{r} \frac{1}{r !}, \quad r=1,2, \ldots, p,
$$

and the polynomial $p$ is uniquely determined if $p \geqslant s-1$. Of course, these are order conditions rather than conditions for $A$-stability. However, in the search for $A$-stable methods it is convenient to start with diagonal elements $b_{11}, b_{22}, \ldots, b_{s s}$, which ensure $A$-stability, and then to solve the (remaining) order conditions. When $p=s$, the diagonal elements must be chosen so that $\alpha_{s}=0$. When $p=s-1$, there is no a priori restriction on the choice of diagonal elements. In some applications that are envisaged, $p=s-2$ is required. Here it suffices to choose both the diagonal elements and $\alpha_{s-1}$, and then to incorporate the choice of $\alpha_{s-1}$ as an extra condition to be satisfied when the order conditions are solved.

Suppose that an $s-1$ stage, semiexplicit, Runge-Kutta method is of order $p=s$, that $b_{r r} \geqslant 0$ for $r=1,2, \ldots, s$, and that at least one diagonal element is zero so that $\beta_{s}=0$. Since $\alpha_{s}=0$, a necessary and sufficient condition for the method to be $A$-stable is that

$$
\sum_{r=[(s+2) / 2]}^{s-1} y^{r} \sum_{j=2 r+1-s}^{r}{ }^{*}\left(\beta_{2 r-j} \beta_{j}-\alpha_{2 r-j} \alpha_{j}\right)(-1)^{r+j} \geqslant 0 \quad \forall y \geqslant 0 .
$$

Some low order cases are now considered.

When $p=s=2$ and $\beta_{2}=0$ all methods are $A$-stable. Since $\alpha_{2}=0$, it follows from (4) that $\beta_{1}=1 / 2$ so that either $b_{11}=1 / 2$ or $b_{22}=1 / 2$.

When $p=s=3$ and $\beta_{3}=0$ a method is $A$-stable if and only if $\beta_{2}^{2} \geqslant \alpha_{2}^{2}$. Since $\alpha_{3}=0$, it follows that $\beta_{2}=\left(\beta_{1}-1 / 3\right) / 2$ and $\beta_{2}^{2}-\alpha_{2}^{2}=\left(\beta_{1}-1 / 2\right) / 6$. Thus, such a method (with nonnegative diagonal elements) is $A$-stable if and only if $\beta_{1} \geqslant 1 / 2$. This inequality is violated if $\beta_{2}=0$, so that there must be at least two nonzero diagonal elements. However, these elements may be chosen equal. An example with $b_{11}=b_{22}$ and $b_{33}=0$ is given by Norsett [5]. An example where $b_{11}=0$ is the method

$$
\begin{array}{c|cccc}
0 & 0 & & & \\
2 c & c & c & c=\frac{3+\sqrt{ } 3}{6} \\
1 & \frac{6 c-1}{12 c} & \frac{1-2 c}{4 c} & c &
\end{array}
$$

When $p=s=4$ and $\beta_{4}=0$ a method is $A$-stable if and only if $\beta_{3}^{2} \geqslant \alpha_{3}^{2}$. Since $\alpha_{4}=0$, it follows from (4) that

$$
\beta_{3}=\frac{1}{2}\left(\beta_{2}-\frac{1}{3} \beta_{1}+\frac{1}{12}\right) \text { and } \beta_{3}^{2}-\alpha_{3}^{2}=\frac{1}{6}\left(\beta_{1}-\frac{1}{2}\right)\left(\beta_{2}-\frac{1}{2} \beta_{1}+\frac{1}{6}\right) \text {. }
$$

As $\beta_{3} \geqslant 0$, necessary and sufficient conditions for $\boldsymbol{A}$-stability are

$$
\beta_{1}-\frac{1}{2} \geqslant 0, \beta_{2}-\frac{1}{2} \beta_{1}+\frac{1}{6} \geqslant 0 \text {. }
$$


When $\beta_{3}=0$, these inequalities give $\beta_{1}=1 / 2$ so that $\beta_{2}=1 / 6$; and the method cannot have real diagonal elements. Thus, there must be at least three nonzero diagonal elements. If these elements are chosen to be equal, then $\beta_{1}=3 c, \beta_{2}=3 c^{2}$ and $\beta_{3}=c^{3}$, and $\alpha_{4}=0$ gives

$$
24 c^{3}-36 c^{2}+12 c-1=0 .
$$

Only the largest root of this equation $c=1.06857902130 \ldots$ satisfies the $A$-stability conditions. An example with $b_{44}=0$ was given in the previous section and is due to Norsett [5]. The case $b_{11}=0$ gives

$$
\begin{array}{c|ccc}
0 & 0 & \\
2 c & c & c & \\
\frac{1+2 c}{2} & \frac{4 c^{2}+8 c-1}{16 c} & \frac{1-4 c^{2}}{16 c} & c \\
1 & \frac{24 c^{2}-12 c+1}{12 c\left(4 c^{2}-1\right)} & \frac{2 c(c-1)}{2 c-1} & \frac{1-6 c}{3 c(2 c+1)} c
\end{array}
$$

An example where the diagonal elements are not equal is the method

$$
\begin{array}{c|cccc}
1 & 1 & & & \\
\frac{1}{2} & -\frac{3}{4} & \frac{5}{4} & & \\
0 & 2 & -3 & 1 & \\
1 & \frac{1}{6} & \frac{2}{3} & \frac{1}{6} & 0
\end{array}
$$

and it is readily verified that the diagonal elements satisfy the $\boldsymbol{A}$-stability conditions.

4. Higher Order Methods. Methods of order $p=5$ present new complications which are not directly associated with the order conditions. Consider the case $p=s$ $=5$ with $\beta_{5}=0$. Then, a method is $A$-stable if and only if

$$
-2\left(\beta_{4} \beta_{2}-\alpha_{4} \alpha_{2}\right)+\beta_{3}^{2}-\alpha_{3}^{2}+y\left(\beta_{4}^{2}-\alpha_{4}^{2}\right) \geqslant 0 \quad \forall y \geqslant 0 .
$$

Since $\alpha_{5}=0$ the condition (4) gives $\beta_{4}=\left(\beta_{3}-\beta_{2} / 3+\beta_{1} / 12-1 / 60\right) / 2$ and hence

$$
\begin{aligned}
& \beta_{4}^{2}-\alpha_{4}^{2}=\frac{1}{6}\left(\beta_{2}-\frac{1}{2} \beta_{1}+\frac{3}{20}\right)\left(\beta_{3}-\frac{1}{2} \beta_{2}+\frac{1}{6} \beta_{1}-\frac{1}{24}\right), \\
& \beta_{3}^{2}-\alpha_{3}^{2}-2\left(\beta_{4} \beta_{2}-\alpha_{4} \alpha_{2}\right)=\frac{1}{6}\left(-\beta_{3}+\frac{1}{2} \beta_{2}-\frac{3}{20} \beta_{1}+\frac{1}{30}\right),
\end{aligned}
$$

so that necessary and sufficient conditions for $A$-stability are that $\beta_{2}-\frac{1}{2} \beta_{1}+\frac{3}{20} \geqslant 0, \quad \beta_{3}-\frac{1}{2} \beta_{2}+\frac{1}{6} \beta_{1}-\frac{1}{24} \geqslant 0, \quad-\beta_{3}+\frac{1}{2} \beta_{2}-\frac{3}{20} \beta_{1}+\frac{1}{30} \geqslant 0$. 
If $\beta_{4}=0$, the first two inequalities give $\beta_{2}-\beta_{1} / 2+3 / 20=0$, whence $\beta_{3}-$ $\beta_{1} / 12+1 / 30=0$. Suppose that $b_{1}, b_{2}$ and $b_{3}$ are the three possible nonzero diagonal elements. If $b_{1}+b_{2}=1 / 2$, the first equation gives $b_{1} b_{2}=1 / 10$ and $b_{1}$ and $b_{2}$ are complex. If $b_{1}+b_{2} \neq 1 / 2$, the equations give

$$
b_{1}^{2}\left(b_{2}^{2}-\frac{1}{2} b_{2}+\frac{1}{12}\right)-b_{1}\left(\frac{1}{2} b_{2}^{2}-\frac{7}{30} b_{2}+\frac{1}{30}\right)+\frac{1}{12} b_{2}^{2}-\frac{1}{30} b_{2}+\frac{1}{240}=0 ;
$$

and $b_{1}$ can be real only if $p(b)=-300 b^{4}+240 b^{3}-84 b^{2}+14 b-1$ is nonnegative for some $b$. If $p(b) \geqslant 0$ for some real $b$, then $p(c) \geqslant 0$ for some $c$ such that $p^{\prime}(c)=0$. But when $p^{\prime}(c)=0$,

$$
p(c)=-\frac{3}{10}\left(20 c^{2}-7 c+1\right)
$$

which is negative for all real $c$. Hence, there must be at least four nonzero diagonal elements in an $A$-stable method with $p=s=5$.

Now suppose that $\beta_{4} \neq 0$ but that the diagonal elements are chosen to be equal. Since $\beta_{4}=\left(\beta_{3}-\beta_{2} / 3+\beta_{1} / 12-1 / 60\right) / 2$, these elements must satisfy

$$
120 c^{4}-240 c^{3}+120 c^{2}-20 c+1=0 .
$$

A direct computation shows that each root violates one of the three inequalities necessary and sufficient for $\boldsymbol{A}$-stability, so that this case differs from the lower order cases. Even when the diagonal elements are not equal it appears that it may not be possible to satisfy the conditions for $A$-stability together with the condition

$$
\beta_{4}=\frac{1}{2}\left(\beta_{3}-\frac{1}{3} \beta_{2}+\frac{1}{12} \beta_{1}-\frac{1}{60}\right) \text {. }
$$

To overcome this it is possible to consider methods where $p=s=5$ but $\beta_{5} \neq 0$. In this case a method is $\boldsymbol{A}$-stable if and only if

$$
\begin{aligned}
2\left(\beta_{5} \beta_{1}-\right. & \left.\alpha_{5} \alpha_{1}\right)-2\left(\beta_{4} \beta_{2}-\alpha_{4} \alpha_{2}\right)+\left(\beta_{3}^{2}-\alpha_{3}^{2}\right) \\
& +y\left\{-2\left(\beta_{5} \beta_{3}-\alpha_{5} \alpha_{3}\right)+\left(\beta_{4}^{2}-\alpha_{4}^{2}\right)\right\}+y^{2}\left(\beta_{5}^{2}-\alpha_{5}^{2}\right) \geqslant 0
\end{aligned}
$$

for all $y \geqslant 0$. Here $\alpha_{5}=0$, where $\alpha_{5}=\beta_{5}-\beta_{4}+\beta_{3} / 2-\beta_{2} / 6+\beta_{1} / 24-1 / 120$. Suppose that all diagonal elements are equal, $b_{11}=b_{22}=\cdots=c$. Then

$$
120 c^{5}-600 c^{4}+600 c^{3}-200 c^{2}+25 c-1=0,
$$

and only one root $c=.278053841136 \ldots$ satisfies the $A$-stability condition (5). It now seems likely that the order conditions (1) cannot be satisfied.

However, it is possible to obtain an $A$-stable method with $p=5$ and $s=6$ with $\beta_{6}=0$. The same $A$-stability condition (5) must be satisfied but it is no longer necessary that $\alpha_{5}=0$. Since $\beta_{5}=0$ implies $\alpha_{5}=0$, it follows as before that there must be at least four nonzero diagonal elements and that these diagonal elements cannot be equal. Hence, suppose that there are five equal diagonal elements so that $\beta_{1}=5 c$, $\beta_{2}=10 c^{2}, \beta_{3}=10 c^{3}$ and $\beta_{4}=5 c^{4}$ and $\beta_{5}=c^{5}$. Although it is now possible to obtain a method where $\alpha_{5}=0$, it is more interesting to consider other values of $c$ which satisfy the $A$-stability condition. In particular, the $A$-stability condition is satisfied if $c=(6-\sqrt{ } 6) / 10 ;$ and this choice gives the following $A$-stable method of order 5: 


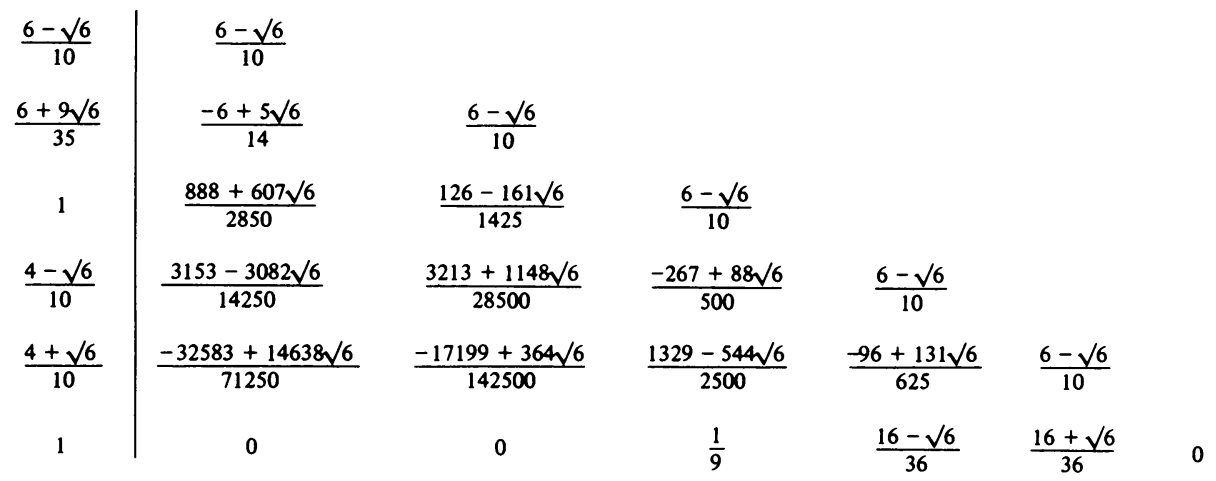

Finally, the case $p=6$ and $s=7$ is considered. When $\beta_{6}=\beta_{7}=0$ the condition for $A$-stability is that

$$
-2\left(\beta_{5} \beta_{3}-\alpha_{5} \alpha_{3}\right)+\left(\beta_{4}^{2}-\alpha_{4}^{2}\right)+y\left(\beta_{5}^{2}-\alpha_{5}^{2}\right) \geqslant 0 \quad \forall y \geqslant 0 ;
$$

and $\alpha_{6}=0$, which gives $\beta_{5}=\left(\beta_{4}-\beta_{3} / 3+\beta_{2} / 12-\beta_{1} / 60+1 / 360\right) / 2$. It follows that necessary and sufficient conditions for $A$-stability are

$$
\begin{gathered}
\beta_{3}-\frac{1}{2} \beta_{2}+\frac{3}{20} \beta_{1}-\frac{1}{30} \geqslant 0, \\
\beta_{4}-\frac{1}{2} \beta_{3}+\frac{1}{6} \beta_{2}-\frac{1}{24} \beta_{1}+\frac{1}{120} \geqslant 0, \\
-\beta_{4} \beta_{1}+\frac{1}{2} \beta_{4}+\frac{1}{2} \beta_{3} \beta_{1}-\frac{4}{15} \beta_{3}-\frac{3}{20} \beta_{2} \beta_{1}+\frac{1}{12} \beta_{2}+\frac{1}{30} \beta_{1}^{2}-\frac{1}{40} \beta_{1}+\frac{1}{288} \geqslant 0 .
\end{gathered}
$$

Suppose that the five nonzero diagonal elements are chosen equal so that $\beta_{1}=5 c, \beta_{2}$ $=10 c^{2}, \beta_{3}=10 c^{3}$ and $\beta_{4}=5 c^{4}$ and $\beta_{5}=c^{5}$. Then, $c$ must satisfy

$$
720 c^{5}-1800 c^{4}+1200 c^{3}-300 c^{2}+30 c-1=0 ;
$$

and there is just one zero $c=.473268391258 \ldots$ which satisfies the conditions. For many problems such a method would require little more computation than that needed for a fifth order $\boldsymbol{A}$-stable method.

To obtain such a method consider a general $s-1$ stage method

$$
\begin{array}{c|cc}
c_{1} & b_{11} & \\
c_{2} & b_{21} & b_{22} \\
\cdot & \cdot & \\
\cdot & \cdot & \\
\cdot & \cdot & \\
c_{s} & b_{s 1} & b_{s 2}
\end{array}
$$

where $c_{r}=b_{r 1}+b_{r 2}+\cdots+b_{r r}$ for $r=1,2, \ldots, s$, and $c_{s}=1$. Let

$$
b_{r}(\sigma)=c_{r}^{\sigma}-\sigma \sum_{j=1}^{r} b_{r j} c_{j}^{\sigma-1}, \quad r=1,2, \ldots, s, \sigma=1,2,3, \ldots,
$$


so that $b_{r}(1)=0$ for $r=1,2, \ldots, s$. Now suppose that $b_{s r} b_{r}(2)=0$ for $r=1,2$,

$\ldots, s$. Then the method is of order $p=6$ if

$$
b_{s}(\sigma)=0, \quad \sigma \leqslant 6,
$$

$$
\sum_{r=1}^{s} b_{s r} c_{r}^{\tau-1} \sum_{i=1}^{s} b_{r i} c_{i}^{\mu-1} b_{i}(\sigma)=0, \quad \sigma+\tau+\mu \leqslant 6
$$

$$
\begin{gathered}
\sum_{r=1}^{s} b_{s r} c_{r}^{\tau-1} \sum_{i=1}^{s} b_{r i} c_{i}^{\mu-1} \sum_{j=1}^{s} b_{i j} c_{j}^{\nu-1} b_{j}(\sigma)=0, \quad \sigma+\tau+\mu+\nu \leqslant 6 \\
\sum_{r=1}^{s} b_{s r} \sum_{i=1}^{s} b_{r i} \sum_{j=1}^{s} b_{i j} \sum_{k=1}^{s} b_{j k} b_{k}(2)=0 \\
\sum_{r=1}^{s} b_{s r} \sum_{i=1}^{s} b_{r i} b_{i}(2) b_{i}(2)=0
\end{gathered}
$$

where $\sigma, \tau, \mu$ and $\nu$ take all possible positive integer values. To simplify these equations assume that

$$
\begin{gathered}
\sum_{r=1}^{s} b_{s r} b_{r j}=b_{s j}\left(1-c_{j}\right), \quad j=3,4, \ldots, s, \\
\sum_{r=1}^{s} b_{s r} c_{r} b_{r j}=\frac{b_{s j}}{2}\left(1-c_{j}^{2}\right), \quad j=3,4, \ldots, s .
\end{gathered}
$$

Provided that $c_{2} \neq c_{1}$ these equations must hold for $j=1,2, \ldots, s$ because

$$
\sum_{r=1}^{s} b_{s r} c_{r}^{\tau-1} b_{r}(1)=0, \quad \sum_{r=1}^{s} b_{s r} c_{r}^{\tau-1} b_{r}(2)=0, \quad \tau=1,2 .
$$

A method with $s=7$ which has the particular form

$$
\begin{array}{l|lllllll}
c & c & & & & & \\
c_{2} & b_{21} & c & & & & \\
c_{3} & b_{31} & b_{32} & c & & & \\
c_{4} & b_{41} & b_{42} & b_{43} & c & & \\
c_{5} & b_{51} & b_{52} & b_{53} & b_{54} & c & & \\
1 & b_{61} & b_{62} & b_{63} & b_{64} & b_{65} & 0 & \\
1 & 0 & 0 & b_{73} & b_{74} & b_{75} & b_{76} & 0
\end{array}
$$


where $c_{r}=b_{r 1}+b_{r 2}+\cdots+b_{r r}$ and $c_{2} \neq c$, is of order $p=6$ if

$$
\begin{gathered}
b_{3}(2)=b_{4}(2)=b_{5}(2)=b_{6}(2)=0, \\
\sum_{r=1}^{s} b_{s r} b_{r j}=b_{s j}\left(1-c_{j}\right), \quad j=3,4,5, \\
\sum_{r=1}^{s} b_{s r} c_{r} b_{r j}=\frac{b_{s j}}{2}\left(1-c_{j}^{2}\right), \quad j=3,4,5, \\
b_{s}(\sigma)=0, \quad \sigma \leqslant 6, \\
\sum_{r=1}^{s} b_{s r} c_{r}^{2} b_{r}(3)=0, \\
\sum_{r=1}^{s} b_{s r} c_{r}^{2} \sum_{i=1}^{s} b_{r i} b_{i}(2)=0 .
\end{gathered}
$$

Since (7.4) implies that $c_{5} \neq 1$ conditions (7.2) and (7.3) give, in particular, $c_{5}=$ $1-2 c$. Condition (7.4) gives the quadrature rule

$$
\int_{0}^{1} p(t) d t=\sum_{r=1}^{s} b_{s r} p\left(c_{r}\right)
$$

valid for any polynomial $p$ of degree 5 . Hence,

$$
\int_{0}^{1}\left(t-c_{3}\right)\left(t-c_{4}\right)\left(t-c_{5}\right)(t-1) d t=0, \int_{0}^{1} t\left(t-c_{3}\right)\left(t-c_{4}\right)\left(t-c_{5}\right)(t-1) d t=0
$$

and these identities imply that $c_{3}$ and $c_{4}$ are the two (real) roots of

$$
5\left(40 c^{2}-24 c+3\right) x^{2}-10\left(16 c^{2}-9 c+1\right) x+20 c^{2}-10 c+1=0 .
$$

The quadrature rule also gives the relations

$$
\begin{aligned}
& b_{s 3}\left(c_{4}-c_{3}\right)\left(c_{5}-c_{3}\right)\left(1-c_{3}\right)=\frac{c_{4} c_{5}}{2}-\frac{c_{4}+c_{5}}{6}+\frac{1}{12}, \\
& b_{s 4}\left(c_{3}-c_{4}\right)\left(c_{5}-c_{4}\right)\left(1-c_{4}\right)=\frac{c_{3} c_{5}}{2}-\frac{c_{3}+c_{5}}{6}+\frac{1}{12}, \\
& b_{s 5}\left(c_{3}-c_{5}\right)\left(c_{4}-c_{5}\right)\left(1-c_{5}\right)=\frac{c_{3} c_{4}}{2}-\frac{c_{3}+c_{4}}{6}+\frac{1}{12}
\end{aligned}
$$

$$
b_{s 6}\left(c_{3}-1\right)\left(c_{4}-1\right)\left(c_{5}-1\right)=\frac{2 c_{5}-1}{2} c_{3} c_{4}-\frac{3 c_{5}-2}{6}\left(c_{3}+c_{4}\right)+\frac{4 c_{5}-3}{12}
$$

which define the quadrature weights.

To obtain an expression for $c_{2}$ consider condition (7.5). Since Eqs. (6.2) must hold, this condition is subsumed by

$$
\sum_{r=1}^{s} b_{s r}\left(c_{r}-1\right)\left(c_{r}-c_{5}\right) b_{r}(\sigma)=0, \quad \sigma=1,2,3,
$$

which give three linear equations. Provided that $c, c_{2}$ and $c_{3}$ are distinct the equations 
give

(8.6) $b_{s 4}\left(c_{4}-1\right)\left(c_{4}-c_{5}\right) b_{41}+b_{s 3}\left(c_{3}-1\right)\left(c_{3}-c_{5}\right) b_{31}=\frac{\alpha_{1} c_{2} c_{3}-\alpha_{2}\left(c_{2}+c_{3}\right)+\alpha_{3}}{\left(c-c_{2}\right)\left(c-c_{3}\right)}$,

(8.7) $b_{s 4}\left(c_{4}-1\right)\left(c_{4}-c_{5}\right) b_{42}+b_{s 3}\left(c_{3}-1\right)\left(c_{3}-c_{5}\right) b_{32}=\frac{\alpha_{1} c c_{3}-\alpha_{2}\left(c+c_{3}\right)+\alpha_{3}}{\left(c_{2}-c\right)\left(c_{2}-c_{3}\right)}$

$$
b_{s 4}\left(c_{4}-1\right)\left(c_{4}-c_{5}\right) b_{43}=\frac{\alpha_{1} c c_{2}-\alpha_{2}\left(c+c_{2}\right)+\alpha_{3}}{\left(c_{3}-c\right)\left(c_{3}-c_{2}\right)}
$$

where $\alpha_{1}, \alpha_{2}$ and $\alpha_{3}$ are given by

$$
\alpha_{1}=\frac{12 c^{2}-8 c+1}{12}, \quad \alpha_{2}=\frac{20 c^{2}-10 c+1}{60}, \quad \alpha_{3}=\frac{30 c^{2}-12 c+1}{180} .
$$

On the other hand, Eqs. (6.3) imply that (7.6) may be replaced by

$$
\sum_{r=1}^{s} b_{s r}\left(c_{r}-1\right)\left(c_{r}-c_{5}\right) \sum_{i=1}^{s} b_{r i} b_{i}(2)=0 \text {. }
$$

Since $b_{r}(2)=0, r=3,4, \ldots, s$, and since $b_{1}(2)=-c^{2}$ and $b_{2}(2)=c_{2}^{2}-4 c c_{2}+2 c^{2}$, this equation gives

$$
\begin{aligned}
b_{s 4}\left(c_{4}-1\right)\left(c_{4}-c_{5}\right) b_{41}+b_{s 3}\left(c_{3}-1\right)\left(c_{3}-c_{5}\right) b_{31} \\
=\frac{c_{2}^{2}-4 c c_{2}+2 c^{2}}{c^{2}}\left\{b_{s 4}\left(c_{4}-1\right)\left(c_{4}-c_{5}\right) b_{42}+b_{s 3}\left(c_{3}-1\right)\left(c_{3}-c_{5}\right) b_{32}\right\} .
\end{aligned}
$$

Equations (8.6) and (8.7) now give a quadratic equation for $c_{2}$ and the root $c_{2}=c$ must be rejected. Thus,

$$
c_{2}=c \frac{c_{3}^{2}\left(2 c \alpha_{1}-3 \alpha_{2}\right)-c_{3}\left(2 c^{2} \alpha_{1}-3 \alpha_{3}\right)+2 c\left(c \alpha_{2}-\alpha_{3}\right)}{c_{3}^{2}\left(c \alpha_{1}-\alpha_{2}\right)-c_{3}\left(2 c^{2} \alpha_{1}-\alpha_{3}\right)+c\left(2 c \alpha_{2}-\alpha_{3}\right)}
$$

Thus, $b_{21}=c_{2}-c$ may be obtained; and since $b_{3}(1)=b_{3}(2)=0$,

$$
b_{32}=\frac{c_{3}^{2}-4 c c_{3}+2 c^{2}}{2\left(c_{2}-c\right)}, \quad b_{31}=c_{3}-c-b_{32} .
$$

Equations (8.6), (8.7) and (8.8) now give $b_{41}, b_{42}$ and $b_{43}$ since the quadrature weights have already been given.

The remaining coefficients may be obtained by considering conditions (7.2) and (7.3). These give

$$
b_{s 6} b_{65}=b_{s 5} c \text {, }
$$

$$
\begin{aligned}
& b_{s 6} b_{64}=-b_{s 4} \frac{c_{4}^{2}+2(3 c-1) c_{4}+4 c^{2}-6 c+1}{4 c} \\
& b_{s 6} b_{63}=-b_{s 3} \frac{c_{3}^{2}+2(3 c-1) c_{3}+4 c^{2}-6 c+1}{4 c}+b_{s 4} b_{43} \frac{c_{5}-c_{4}}{2 c},
\end{aligned}
$$




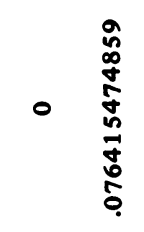

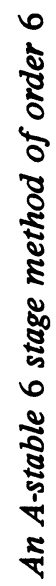
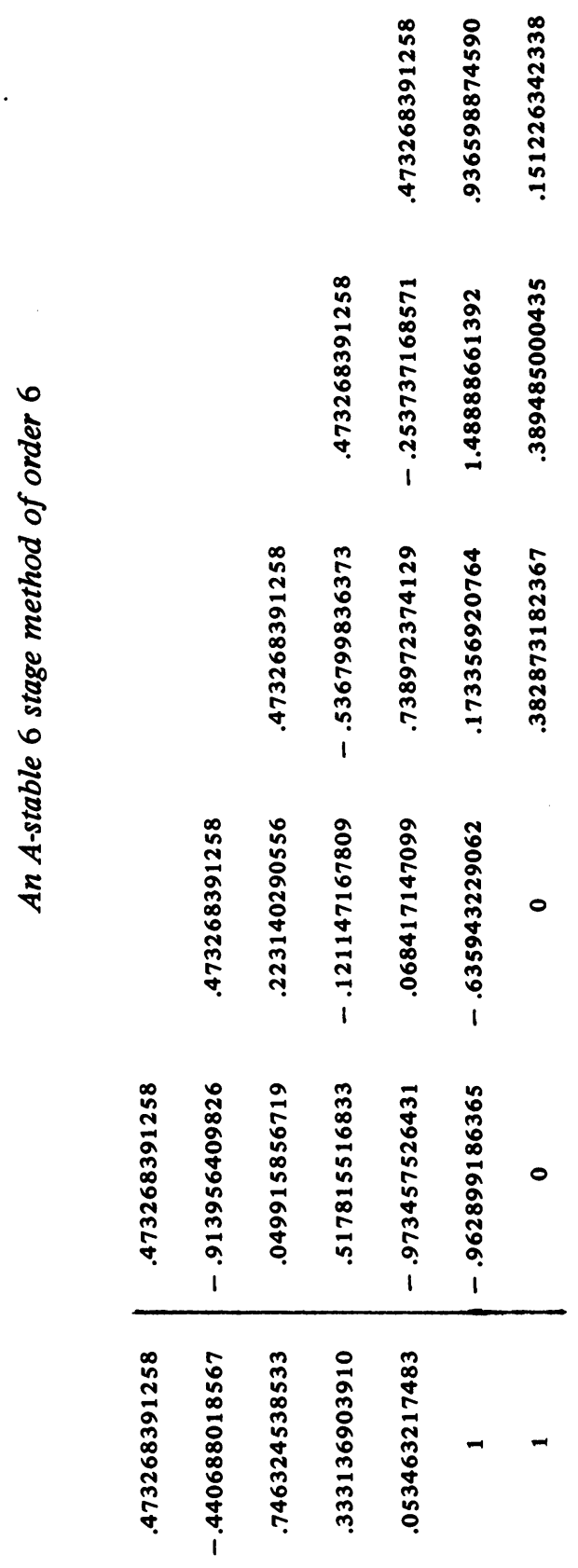


$$
b_{s 5} b_{54}=b_{s 4} \frac{\left(1-c_{4}\right)\left(c_{5}-c_{4}\right)}{4 c}
$$

$$
b_{s 5} b_{53}=b_{s 3} \frac{\left(1-c_{3}\right)\left(c_{5}-c_{3}\right)}{4 c}-b_{s 4} b_{43} \frac{1-c_{4}}{2 c} \text {. }
$$

Finally, $b_{5}(1)=b_{5}(2)=0$ and $b_{6}(1)=b_{6}(2)=0$ give

$$
\begin{aligned}
& b_{52}=\frac{14 c^{2}-8 c+1+2 b_{53}\left(c-c_{3}\right)+2 b_{54}\left(c-c_{4}\right)}{2\left(c_{2}-c\right)}, \\
& b_{62}=\frac{c_{5}+2 b_{63}\left(c-c_{3}\right)+2 b_{64}\left(c-c_{4}\right)+2 b_{65}\left(c-c_{5}\right)}{2\left(c_{2}-c\right)},
\end{aligned}
$$

and $b_{51}=c_{5}-c-b_{52}-b_{53}-b_{54}$ and $b_{61}=1-b_{62}-b_{63}-b_{64}-b_{65}$.

Because $c_{3}$ and $c_{4}$ are the roots of the quadratic equation (8.1) there are no simple expressions for the coefficients in terms of $c$ alone. However, the coefficients are easily computed from Eqs. (8) once $c$ has been obtained as the root $c=.473268391258$ $\ldots$ of $720 c^{5}-1800 c^{4}+1200 c^{3}-300 c^{2}+30 c-1$. The coefficients have been computed to 20 significant decimal figures and the results, rounded to 12 decimal figures, are given in the table. Each author has computed these coefficients independently, and it has been verified that the coefficients satisfy all the order conditions given by Butcher [1]. A similar method may be obtained by interchanging $c_{3}$ and $c_{4}$ and this gives $c_{2} \in(0,1)$, but some coefficients are large.

School of Mathematical and Physical Sciences

University of Sussex

Brighton, BN1 9QH, England

Mathematics Department

College of Science

University of Baghdad

Baghdad, Iraq

1. J. C. BUTCHER, "Coefficients for the study of Runge-Kutta integration processes," $J$. Austral. Math. Soc., v. 3, 1963, pp. 185-201.

2. J. C. BUTCHER, "On the implementation of implicit Runge-Kutta methods," BIT, v. 16, 1976, pp. $237-240$.

3. G. J. COOPER \& J. H. VERNER, "Some explicit Runge-Kutta methods of high order," SIAM J. Numer. Anal., v. 9, 1972, pp. 389-405.

4. G. G. DAHLQUIST, "A special stability problem for linear multistep methods," $B I T, \mathrm{v}$. 3, 1963, pp. 27-43.

5. S. P. NORSETT, Semi-Explicit Runge-Kutta Methods, Mathematics Department, University of Trondheim, Reprint No. 6/74.

6. S. P. NORSETT, "C-polynomials for rational approximation to the exponential function," Numer. Math., v. 25, 1975, pp. 39-56. 